\title{
Cavin-2 Functions as a Suppressive Regulator in TNF-induced Mesenchymal Stromal Cell Inflammation and Angiogenic Phenotypes
}

\author{
Bayader Annabi, ${ }^{1,2}$, Alain Zgheib ${ }^{1}$, Borhane Annabi ${ }^{1}$ \\ ${ }^{1}$ Laboratoire d'Oncologie Moléculaire, Département de Chimie, Centre de recherche BIOMED, Université du Québec à Montréal, Quebec, \\ ${ }^{2}$ Département de Physiologie Moléculaire et Intégrative, Faculté de Médecine, Université de Montréal, Montreal, Canada
}

Tumour necrosis factor (TNF)- $\alpha$ activation of mesenchymal stromal cells (MSC) enhances their tumour-suppressive properties and tumour-homing ability. The molecular actors involved are unknown. We found that TNF induced MSC migration and tubulogenesis which correlated with a dose-dependent increase in Cavin-1 and Cavin-3 transcript levels. TNF triggered cyclooxygenase (COX)-2 expression, whereas specific siRNA-mediated gene silencing of Cavin-2 resulted in an amplified COX-2 expression, tubulogenesis, and migratory response partially due to a rapid and sustained increase in NF- $\kappa \mathrm{B}$ phosphorylation status. Our results highlight a suppressive role for the caveolar component Cavin-2 in the angiogenic and inflammatory regulation of TNF-activated MSC.

Keywords: Mesenchymal stromal cells, Cavin, TNF, Cancer, Angiogenesis, Inflammation

\section{Introduction}

Mesenchymal stromal cells (MSC), most commonly isolated from the bone marrow $(1,2)$, are a population of pluripotent adult stem cells that have been shown to be recruited within experimental vascularizing tumours (3-5). This incorporation of MSC within a pro-inflammatory tumour architecture, combined with intrinsic immunomodulatory mechanisms, implies that they must also respond to tumour-derived growth factor or cytokine cues $(5,6)$.

\footnotetext{
Accepted for publication November 24, 2016, Published online May 30, 2017

Correspondence to Borhane Annabi

Département de Chimie, Université du Québec à Montréal, C.P. 8888, Succ. Centre-ville, Montréal, Québec, Canada, H3C 3P8

Tel: +514-987-3000 ext 7610, Fax: +514-987-0246

E-mail: annabi.borhane@uqam.ca

(c) This is an open-access article distributed under the terms of the Creative Commons Attribution Non-Commercial License (http://creativecommons.org/ licenses/by-nc/4.0/), which permits unrestricted non-commercial use, distribution, and reproduction in any medium, provided the original work is properly cited.

Copyright (c) 2017 by the Korean Society for Stem Cells Research
}

The homing of MSC to tumours is therefore believed to be among the earliest phenomena involving MSC-cancer interactions, as was recently reported in a mouse model where injected human MSC preferentially migrated towards implanted human melanoma tumours (3). Subsequent studies have further confirmed MSC homing to tumours and to sites of metastasis (7), and cotransplantation of MSC with melanoma cells in mice was found to enhance tumour engraftment and growth (8). More recently, MSC were reported to migrate to the tumour parenchyma and to differentiate into pericytes, inducing tumour vasculogenesis and promoting tumour recurrence (9). MSC were also found to promote tumour growth both in vitro and in vivo, suggesting that tumour promotion may be partially attributable to enhanced angiogenesis (10), and through interactions with endothelial cells (11). Although some molecular partners have been identified, most of the signal transducing contributors and the subcellular compartments involved still remain to be determined.

Multiple cytokine-mediated signaling cascades are compartimentalized within caveolae, which represent specialized cholesterol- and sphingolipid-enriched plasma mem- 
brane invaginations, and which, in cancer cells, regulate oncogenic functions (12). Among these functions, roles have been described for caveolae in mechanosensing, vesicular trafficking, and shuttling of molecules through the cell $(13,14)$. Caveolae have also been ascribed functions in many physiological events $(15,16)$, and in pathological settings such as cancer progression and metastasis (17). Cancer cell invasion, which involves several cellular processes including disruption of cell adhesion, degradation of the extracellular matrix (ECM) and regulation of cell invasion through blood and lymph vessels, has also been shown, in part, to require caveolae (18). Furthermore, the clustering of signaling molecules within these cholesterol-rich membrane microdomains has already revealed a role for Caveolin-1 in osteogenic differentiation of MSC $(19,20)$. It is, however, unknown whether alternate caveolar components are involved in the regulation of the angiogenic and inflammation phenotypes of MSC.

Coat proteins called Cavins have recently been suggested not only to work together with Caveolins to regulate the formation of caveolae, but also to have the potential to transmit signals dynamically from caveolae in response to angiogenic or inflammatory cues (21-23). Of the four Cavin proteins, PTRF/Cavin-1 has been shown to mediate reduced production of the pro-inflammatory biomarker MMP-9, independent of caveolar formation, and has been reported to decrease metastatic PC3 prostate cancer cell migration (24). Interestingly, MMP-9 transcriptional regulation is already considered to be triggered by cytokines, such as tumour necrosis factor (TNF)- $\alpha$, through transcription factors including SP-1, AP-1 and NF- $\kappa \mathrm{B}$ (25). Additionally, several studies have pointed out dual roles for other Cavin proteins (26), including Cavin-2 (SDPR, for serum deprivation protein response), Cavin-3 (SRBC, as an Sdr-related gene product that binds to c-kinase), and Cavin-4 (MURC, for muscle restricted coiled-coil protein). Most notably, a decrease in Cavin-2 expression is known to lead to decreased caveolar formation and numbers, as it co-localizes at the plasma membrane along with Caveolin-1 and Cavin-1 (27). Cavin-3 was first identified as a substrate of PKC delta and is located in a tumour suppressor region at chromosome $11 \mathrm{p} 15.5$, which is lost in breast, prostate and pancreatic cancer cells (28). Cavin-4 expression is restricted to smooth muscle, such as skeletal and cardiac myocytes (29).

In line with recent evidence which indicated an elevated inflammatory response in Caveolin-1-deficient mice (30), one may envision that caveolae, in fact, exert some repressive functions upon inflammatory cues. In addition, Caveolin-1 KO mice exhibit upregulated transcriptional control of inflammatory cytokines including IL-6 and TNF- $\alpha$ (30). Whether any of the Cavin proteins contribute to such repressive control and could thus impact the TNF-mediated inflammatory or angiogenic phenotype of MSC is unknown. In the current study, we examined which of the Cavin family members could alter the TNF-mediated response in MSC.

\section{Materials and Methods}

\section{Materials}

Tumour necrosis factor (TNF)- $\alpha$ was from EMD Millipore (Gibbstown, NJ), sodium dodecylsulfate (SDS) and bovine serum albumin (BSA) were purchased from Sigma (Oakville, ON). Electrophoresis reagents were purchased from Bio-Rad (Mississauga, ON). The enhanced chemiluminescence (ECL) reagents were from Perkin Elmer (Waltham, MA). Micro bicinchoninic acid protein assay reagents were from Pierce (Rockford, IL). The polyclonal antibodies against $\mathrm{I} \kappa \mathrm{B}$ and phosphorylated $\mathrm{I} \kappa \mathrm{B}$ were from Cell Signaling (Danvers, MA). The polyclonal anti-Cavin-1 antibody (ABE1953) was from EMD Millipore (Etobicoke, ON), whereas the anti-Cavin-2 and anti-Cavin-3 antibodies were from ABNOVA (Walnut, CA). The monoclonal antibody against GAPDH was from Advanced Immunochemical Inc. (Long Beach, CA). The anti-COX-2 antibody (610203) was from BD Biosciences (San Jose, CA). Horseradish peroxidase-conjugated donkey anti-rabbit and anti-mouse IgG secondary antibodies were from Jackson ImmunoResearch Laboratories (West Grove, PA). All other reagents were from Sigma-Aldrich Canada.

\section{Cell cultures}

Human MSC were obtained from marrow biopsies of volunteers undergoing hip replacement and were isolated by Ficoll gradient. Cells were plated in high glucose Dulbecco's modified Eagle's medium (DMEM; GibcoBRL) supplemented with $10 \%$ inactivated fetal bovine serum (iFBS) (Hyclone Laboratories, Logan, UT) and 100 units/ml Penicillin/Streptomycin. After 5 7 days of incubation in a humidified incubator at $37^{\circ} \mathrm{C}$ with $5 \% \mathrm{CO}_{2}$, the nonadherent hematopoietic cells were discarded. Adherent MSC were further grown on non-coated culture dishes and maintained over 14 passages. Analysis by flow cytometry performed at passage 14 revealed that MSC expressed CD44, yet were negative for CD45, CD31, KDR/flk1 (VEGF-R2), flt-4 (VEGF-R3), and Tie2 (angiopoietin receptor) (data not shown). Serum starvation was performed by culturing the cells in high $\alpha$ MEM, $2 \mathrm{mM}$ 
L-glutamine, and 100 units/ml Penicillin/Streptomycin from which the inactivated fetal bovine serum was omitted.

\section{Immunoblotting procedures}

Following treatment or transfection, MSC were washed with PBS and lysed with lysis buffer $(50 \mathrm{mM}$ Tris- $\mathrm{HCl}$, pH 7.4, $120 \mathrm{mM} \mathrm{NaCl,} 5 \mathrm{mM}$ EDTA, 0.5\% Nonidet P-40, $0.1 \%$ Triton) in the presence of phosphatase and protease inhibitors on ice for 30 minutes. Cell debris was pelleted by centrifugation for $10 \mathrm{~min}$ at high speed. Protein concentration was quantified using a micro bicinchoninic acid protein assay kit (Thermo Fisher Scientific Inc). Proteins from control and treated cells were separated by sodium dodecyl sulfate (SDS)-polyacrylamide gel electrophoresis (PAGE). After electrophoresis, proteins were electrotransferred to polyvinylidene difluoride membranes, which were then blocked overnight at $4^{\circ} \mathrm{C}$ with $5 \%$ non-fat dry milk in Tris-buffered saline $(150 \mathrm{mM} \mathrm{NaCl}, 20 \mathrm{mM}$ Tris-HCl, $\mathrm{pH} 7.5$ ) containing $0.3 \%$ Tween-20 (TBST). Membranes were further washed in TBST and incubated with primary antibodies directed against COX-2 (1/10,000), $\mathrm{I} \kappa \mathrm{B}$, phosphorylated $\mathrm{I} \kappa \mathrm{B}(1 / 1,000)$, or GAPDH $(1 / 1,500)$. Washing was then performed in TBST, followed by a 1 hour incubation with horseradish peroxidase-conjugated anti-rabbit $\operatorname{IgG}(1 / 10,000)$ or anti-mouse $\operatorname{IgG}(1 / 5,000)$ in TBST containing 5\% non-fat dry milk. Immunoreactive material was visualized by Western Lightning Enhanced Chemiluminescence Pro reagents (Perkin Elmer).

\section{Real-time cell migration assay}

Cell migration assay experiments were carried out using the Real-Time Cell Analyzer (RTCA) Dual-Plate (DP) Instrument of the xCELLigence system (Roche Diagnostics). MSC were trypsinized and 20,000 cells/well were seeded onto CIM-Plates 16 (Roche Diagnostics). These plates are similar to conventional Transwells ( $8 \mu \mathrm{m}$ pore size) but with gold electrode arrays on the bottom side of the membrane to provide a real-time measurement of cell migration. Prior to cell seeding, the underside of the wells from the upper chamber was coated with $25 \mu \mathrm{L}$ of $0.15 \%$ gelatin in PBS and incubated for 1 hour at $37^{\circ} \mathrm{C}$. TNF was dissolved in serum-free culture medium and added to the upper wells, while the lower chamber was filled with $10 \%$ Fetal Calf Serum medium to act as a chemoattractant. Cell migration was monitored for 8 hours. The impedance values were measured by the RTCA DP Instrument software and were expressed in arbitrary units as the Relative Cell Migration Index. Each experiment was performed in triplicate.

\section{Total RNA isolation, cDNA synthesis and real-time quantitative RT-PCR}

Total RNA was extracted from MSC monolayers using TRIzol reagent (Life Technologies, Gaithersburg, MD). For cDNA synthesis, $1 \mu \mathrm{g}$ of total RNA was reverse-transcribed into cDNA using a high capacity cDNA reverse transcription kit (Applied Biosystems, Foster City, CA). cDNA was stored at $-80^{\circ} \mathrm{C}$ prior to PCR. Gene expression was quantified by real-time quantitative PCR using iQ SYBR Green Supermix (BIO-RAD, Hercules, CA). DNA amplification was carried out using an Icycler iQ5 (BIO-RAD, Hercules, CA) and product detection was performed by measuring binding of the fluorescent dye SYBR Green I to double-stranded DNA. The following primer sets were provided by QIAGEN (Valencia, CA): Cavin-1 (Hs_CAV1_1_SG, QT00001533), Cavin-2 (Hs_CAV2_1_SG, QT00068754), Cavin-3 (Hs_CAV3_1_SG, QT00001414), $\beta$-Actin (Hs_ACTB_2_SG, QT01680476), GAPDH (Hs GAPDH_1_SG, QT00079247), PPIA (Hs_PPIA_4_SG, QT01866137). The relative quantities of target gene mRNA against an internal control, $\beta$-Actin/GAPDH/PPIA RNA, were measured by following a $\Delta \mathrm{C}_{\mathrm{T}}$ method employing an amplification plot (fluorescence signal $v$ s. cycle number). The difference $\left(\Delta \mathrm{C}_{\mathrm{T}}\right)$ between the mean values in the triplicate samples of target gene and those of $\beta$-Actin/GAPDH/PPIA RNA were calculated by iQ5 Optical System Software version 2.0 (BIO-RAD, Hercules, CA) and the relative quantified value $(\mathrm{RQV})$ was expressed as $2^{-\Delta \mathrm{C}}$. Semi-quantitative PCR was performed to examine amplification products and amplicons resolved on 1.8\% agarose gels containing $1 \mu \mathrm{g} / \mathrm{ml}$ ethidium bromide.

\section{Transfection method and RNA interference}

Subconfluent (30 50\%) MSC were transiently transfected for 24 hours in serum-free media and with $20 \mathrm{nM}$ siRNA against Cavin-1 (human Hs_CAV1_7 FlexiTube siRNA, SI02662338), Cavin-2 (human Hs_CAV2_6 HP siRNA, SI03648841), Cavin-3 (HS_CAV3_5 FlexiTube siRNA, SI02223221), or scrambled sequences (AllStar Negative Control siRNA, 1027281) using Lipofectamine 2000 transfection reagent (Invitrogen, CA). Small interfering RNA and mismatch siRNA were synthesized by QIAGEN and annealed to form duplexes.

\section{Capillary-like structure formation assay}

Induction of tubulogenesis was performed using Matrigel. Matrigel was thawed on ice to prevent premature polymerization; aliquots of $50 \mu 1$ were plated into individual wells of 96-well tissue culture plates (Costar) and allowed to polymerize at $37^{\circ} \mathrm{C}$ for at least 60 minutes. Cells were re- 
moved from confluent cultures by treatment with $0.05 \%$ trypsin, $0.53 \mathrm{mM}$ EDTA. The cells were washed in serum-containing medium then resuspended to $10^{6}$ cells $/ \mathrm{ml}$. Each culture well received $100-\mu 1$ of the cell suspension with or without $100 \mu \mathrm{M}$ TNF. Each control or TNF treatment was assayed in duplicate, and all experiments were performed at least three times. For quantitation of tube formation, the total length of the tubes formed in a unit area was digitized and measured using ECLIPSE software. For each test, five randomly chosen areas were measured and averaged.

\section{Statistical data analysis}

Data are representative of three or more independent experiments. Statistical significance was assessed using Student's unpaired $t$-test. Probability values of less than 0.05 were considered significant and an asterisk $\left(^{\star}\right)$ identifies such significance in the figures.

\section{Results}

\section{TNF triggers a global MSC angiogenic phenotype by inducing cell migration and tubulogenesis}

We first assessed the effect of TNF treatment on MSC's angiogenic phenotype using both a real-time cell migration assay as well as measuring the cells ability to generate a three-dimentional capillary-like structure formation (tubulogenesis) on Matrigel. We found that the cell migration response to TNF was increased in a dose-dependent manner (Fig. 1A). When data were compared after 8 hours, cell migration tended to plateau between $30 \sim 100$ $\mu \mathrm{M}$ TNF (Fig. 1B) and it was decided to maintain the $\mathrm{TNF}$ at $100 \mu \mathrm{M}$ for all subsequent experiments in order to correlate the molecular signatures associated with an optimal TNF-mediated cellular effect. We have previously observed that MSC possess intrinsic angiogenic properties since they can generate capillary-like structures in vitro, and that this process was regulated at the molecular level in part through a specific membrane-bound MMP, namely membrane-type (MT)1-MMP and by sphingosine-1-phosphate, which are both involved in tumour angiogenesis $(31,32)$. Increasing quantities of MSC were therefore seeded onto Matrigel and tubulogenesis was assessed within 6 hours as described in the Methods section. We found that significant tubulogenesis was observed when 40,000 80,000 cells were seeded (Fig. 1C), reached a plateau at 100,000 cells, and that TNF augmented this process (Fig. 1D). The potential interrelationship between the MSC angiogenic phenotype and Cavin expression was next assessed.
TNF triggers Cavin-1 and Cavin-3 transcription, while Cavin-2 transcripts are decreased

Cavin gene and Cavin protein expression profiling was next performed using respectively total RNA or total cell lysates isolated from control and TNF-treated MSC. We found that each of the Cavin-1, Cavin-2, and Cavin-3 transcripts were expressed in MSC because single specific amplicons were generated upon RT-qPCR and visualized on an agarose gel (Fig. 2A). Cavin-1 and Cavin-3 transcript levels were dose-dependently increased in response to TNF, whereas that of Cavin-2 decreased upon TNF treatment (Fig. 2B). Cavin-1, -2, and -3 protein levels were assessed by Western blotting (Fig. 2C) and confirmed TNF-mediated increases in Cavin-1 and -3, whereas Cavin-2 (Fig. 2C, arrow) levels decreased (Fig. 2D). Altogether, our data confirm that TNF triggers an angiogenic phenotype in MSC by increasing cell migration and tubulogenesis (Fig. 1), and that it is able to differentially modulate Cavin expression in MSC. We next sought to investigate the potential impact of Cavins on the TNF response.

\section{Silencing of Cavin-2, but not Cavin-1 or -3 , potentiates TNF-mediated COX-2 expression}

MSC were next transiently transfected with siRNA in order to silence Cavin-1, -2, or -3 gene expression. Gene silencing specificity was validated by qRT-PCR, and found to effectively reduce gene expression between $75 \sim 90 \%$ (Fig. 3A). When cyclooxygenase (COX)-2, a pro-inflammatory biomarker known to be induced by TNF, expression was assessed, we found that neither Cavin-1 nor Cavin-3 gene silencing affected TNF-induced COX-2 expression (Fig. 3B). In contrast, silencing Cavin-2 was found to potentiate TNF-mediated COX-2 induction (Fig. 3B). Our observations suggest that Cavin-2 exerts a pivotal role in TNF-mediated signalling in MSC as its expression represses basal TNF signalling.

\section{Silencing of Cavin-2, but not Cavin-1 or -3 , potentiates TNF-mediated MSC migration and tubulogenesis}

When MSC migration was asessed, none of the three Cavin gene silencings altered basal migration (Fig. 4A, open circles). TNF-mediated MSC migration was induced in siScrambled, siCavin-1 and in siCavin-3 transfected cells, although to a lesser extent than in untransfected cells, due to transfection conditions, whereas it was potentiated in the MSC where Cavin-2 gene expression was reduced (Fig. 4A, closed circles). Given that TNF is able to increase MSC migration and that Cavin-2 regulates this 

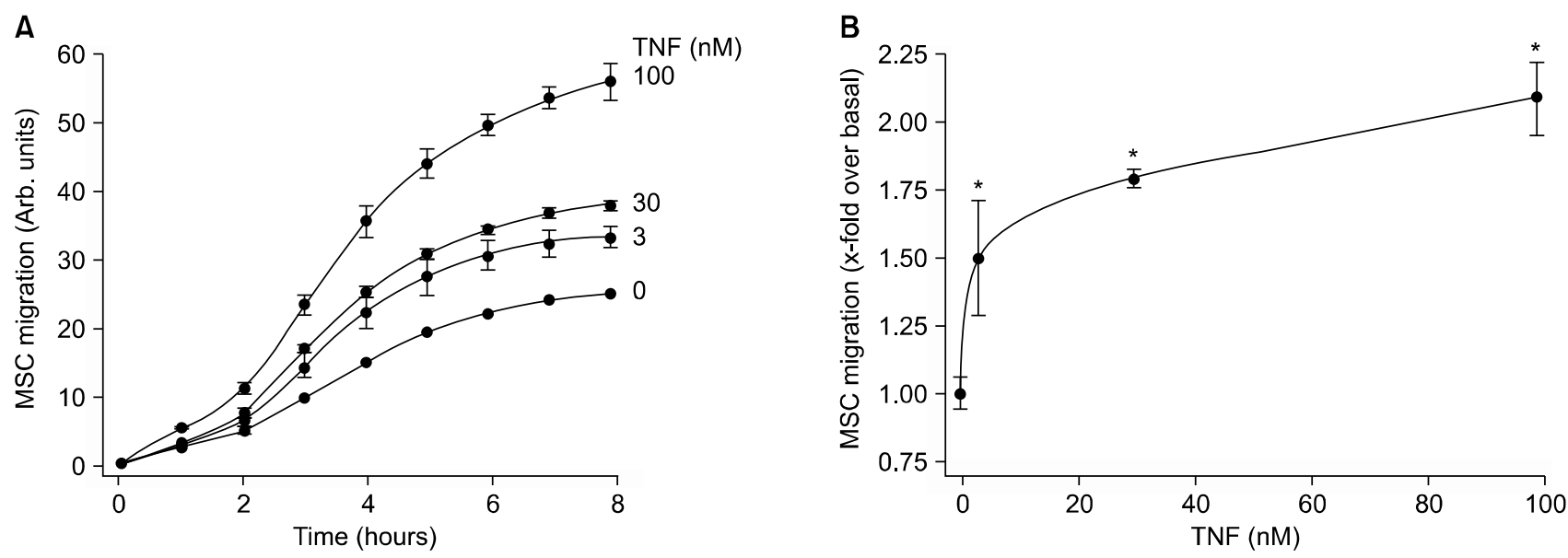

C

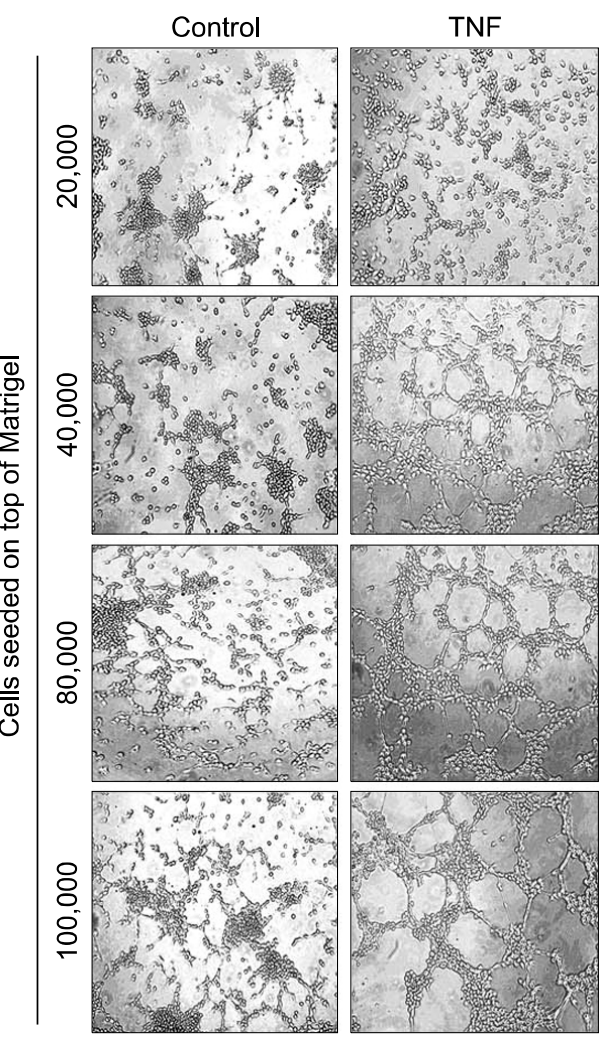

D

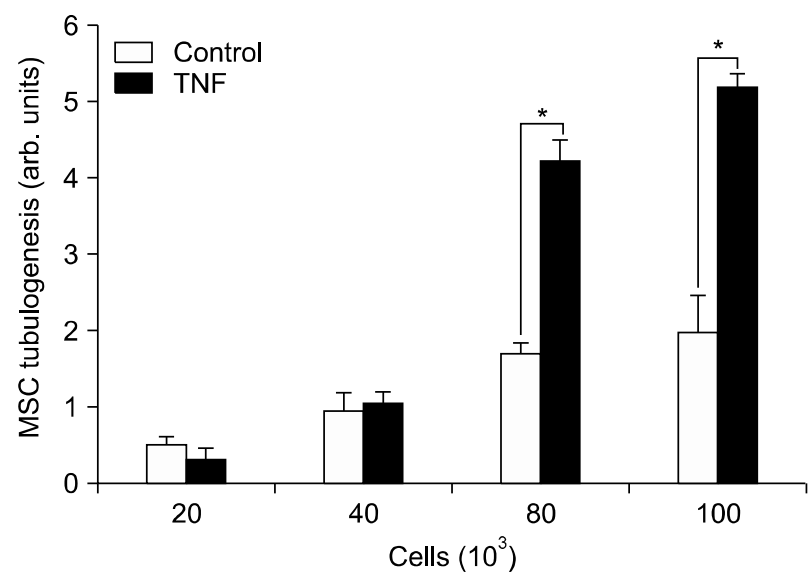

Fig. 1. TNF triggers a global MSC angiogenic phenotype by inducing cell migration and tubulogenesis. (A) MSC were harvested and the rate of cell migration monitored in real-time in response to various TNF concentrations using the xCELLigence system. Representative plots from two experiments measuring impedance responses are shown for each condition. (B) Plot of cell migration at time $=8$ hours is shown in response to each TNF concentration used. (C) Various quantities of MSC were seeded on top of Matrigel and tubulogenesis was allowed to proceed for 6 hours as described in the Methods section. (D) Quantification of the 3D-capillary-like structures was performed as described in the Methods section for control (open box) and $100 \mathrm{nM}$ TNF-treated cells (closed box). Data is from a representative experiment, and each point is the mean of 5 randomly chosen areas.

response, we next examined whether tubulogenesis was also altered. When Cavin-2 gene expression was silenced and tubulogenesis measured at a sub-saturating cell density (40,000 cells), we found that TNF, similar to its effect on cell migration and COX-2 expression, showed greater amplification of MSC tube formation as compared to control (siScrambled) cells (Fig. 4B). 
A
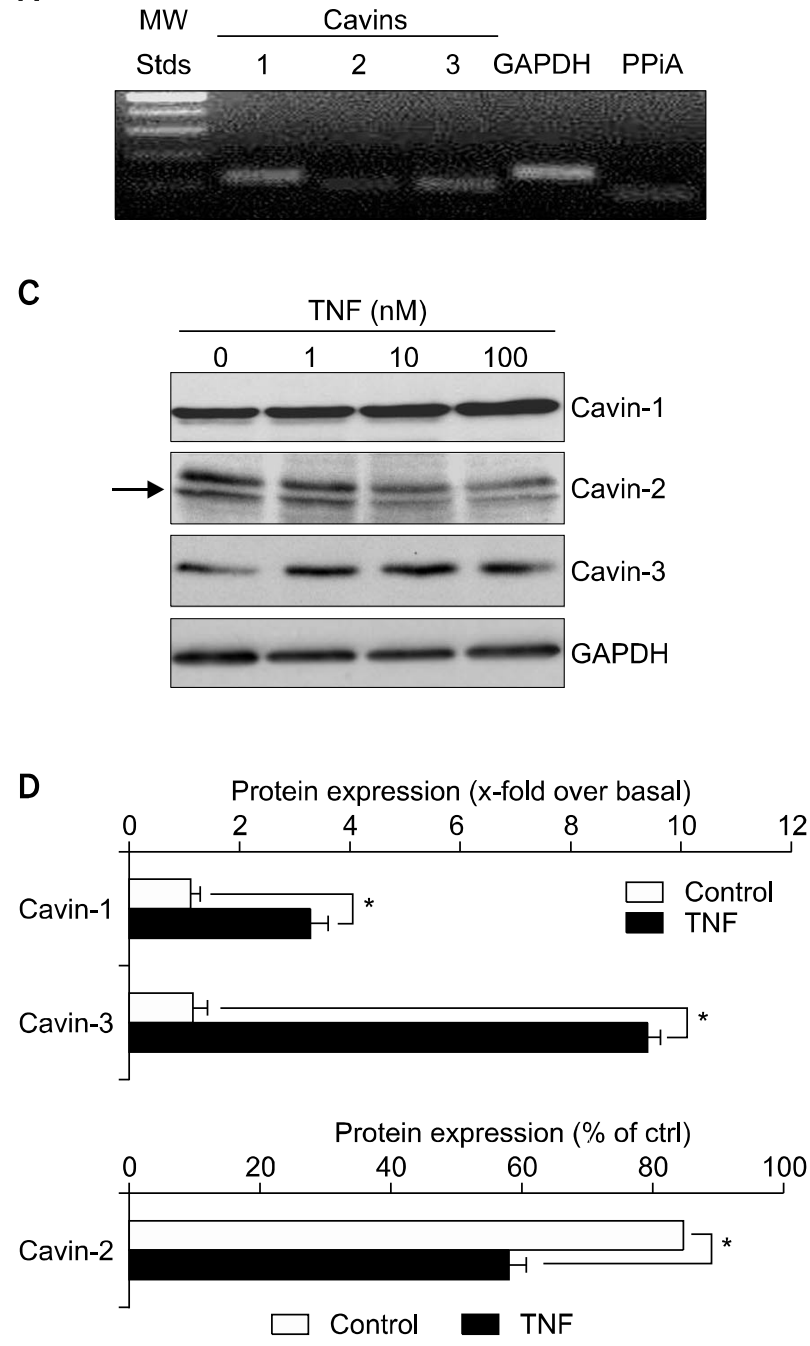

B
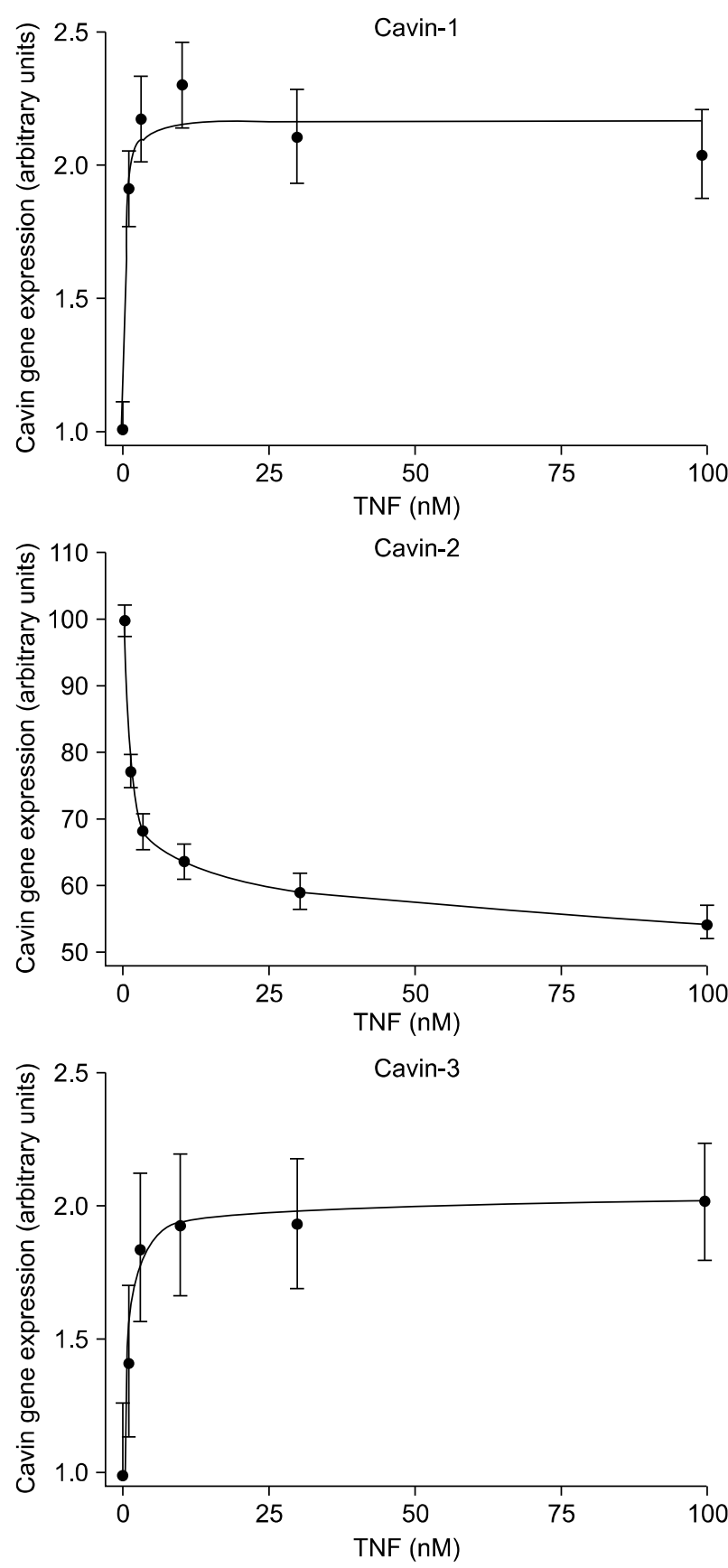

Fig. 2. TNF triggers Cavin-1 and Cavin-3 transcription, while Cavin-2 transcripts are decreased. Total RNA was extracted from MSC and RT-qPCR performed as described in the Methods section. (A) Amplicons of Cavins were migrated on agarose gels and each showed a single product. GAPDH, glyceraldehyde 3-phosphate dehydrogenase; PPIA, peptidyl prolylisomerase A. (B) Cavin-1, Cavin-2, and Cavin-3 gene expression and $(C)$ protein expression were assessed in serum-starved MSC following 24 hours treatment with the indicated TNF concentrations. (D) Protein levels were assessed by scanning densitometry in vehicle (white bars) and $100 \mathrm{nM}$ TNF-treated (black bars) MSC.

\section{TNF-mediated NF- $\kappa$ B signaling occurs rapidely and is sustained upon Cavin-2 gene silencing}

In order to investigate the possible signal transducing mechanisms responsible for increased TNF-induced MSC migration, tubulogenesis and COX-2 expression upon Cavin-2 silencing, we decided to examine the phosphorylation status of $\mathrm{I} \kappa \mathrm{B}$ and its subsequent proteasomal-mediated degradation. MSC were transiently-transfected with 
A

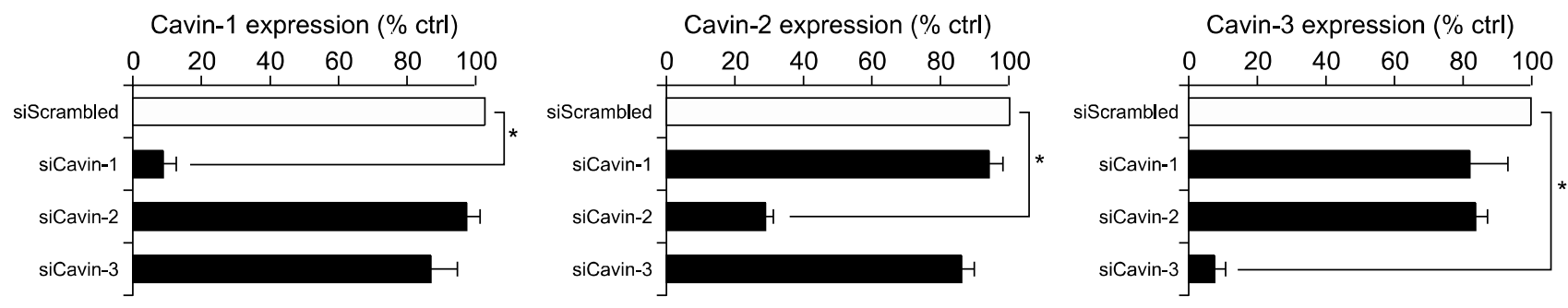

B

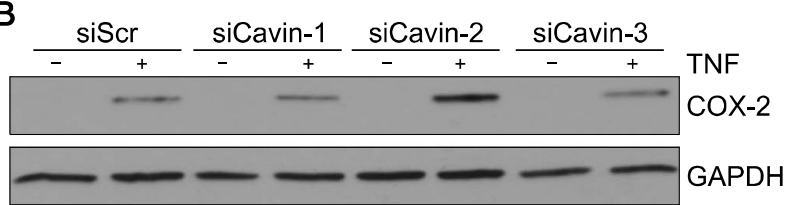

Fig. 3. Silencing of Cavin-2, but not Cavin-1 or -3 , potentiates TNF-mediated COX-2 expression. MSC were transiantly transfected with specific siRNA against each of the Cavin-1, -2 , or -3 genes as described in the Methods section. (A) Total RNA was extracted and Cavin gene silencing efficacy and specificity were assessed by qRT-PCR. (B) MSC were treated with $100 \mathrm{nM}$ TNF for 24 hours and COX-2 expression was immunodetected in lysates as described in the Methods section. GAPDH expression served as a loading control.
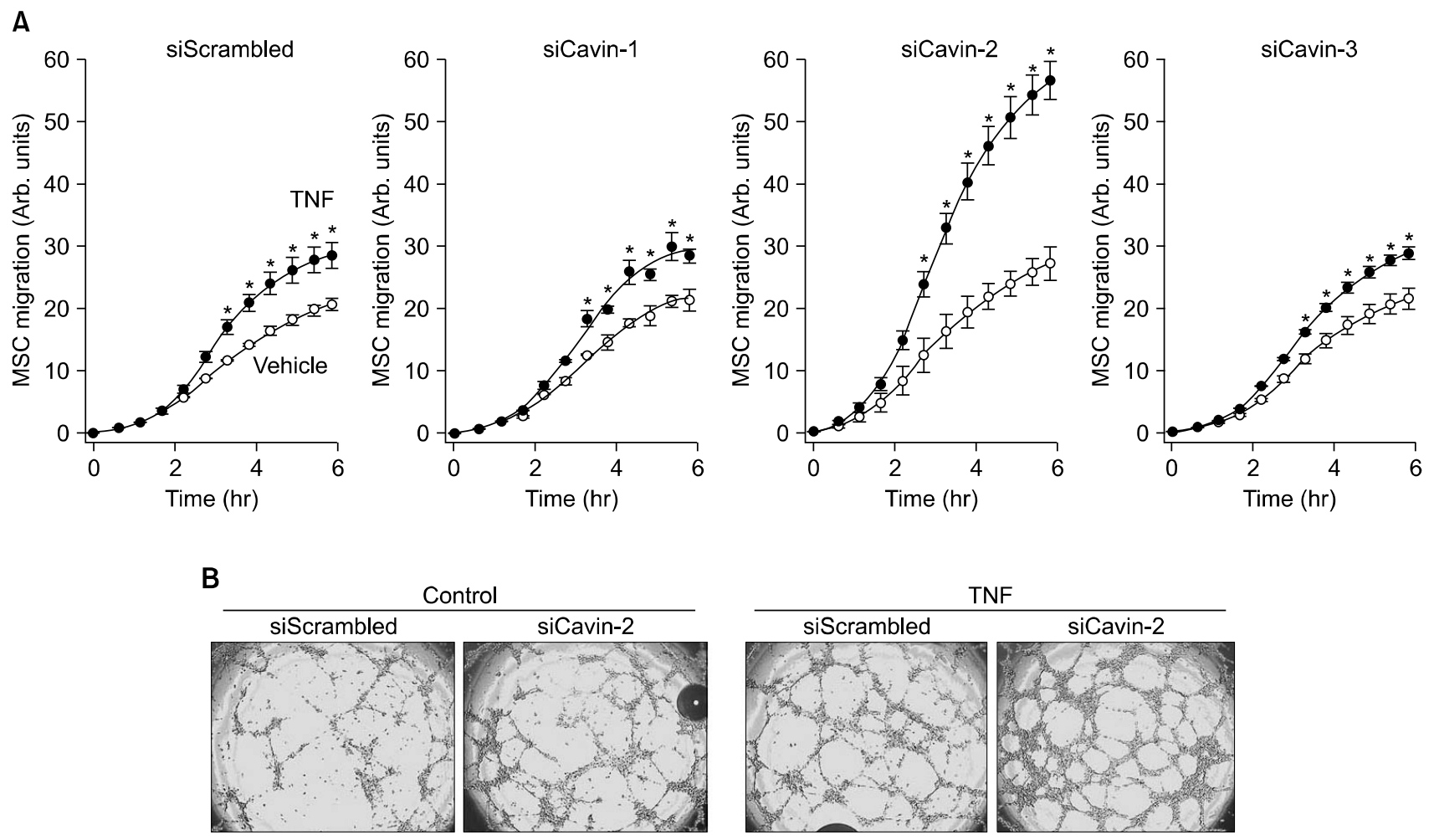

Fig. 4. Silencing of Cavin-2, but not Cavin-1 or -3, potentiates TNF-mediated MSC migration and tubulogenesis. MSC were transiently transfected with specific siRNA against each of the Cavin-1, -2, or -3 genes as described in the Methods section. (A) Transfected-MSC were harvested and cell migration in response to vehicle (open circles) or to $100 \mathrm{nM}$ TNF (closed circles) was assessed as described in the Methods section. (B) Transient gene silencing was performed in control (siScrambled) and in siCavin-2-transfected MSC as described in the Methods section. Cells were then left to recuperate, trypsinized and 40,000 cells were seeded on top of Matrigel. A tubulogenesis assay was performed as described in the Methods section and images were taken at 6 hours. 
A
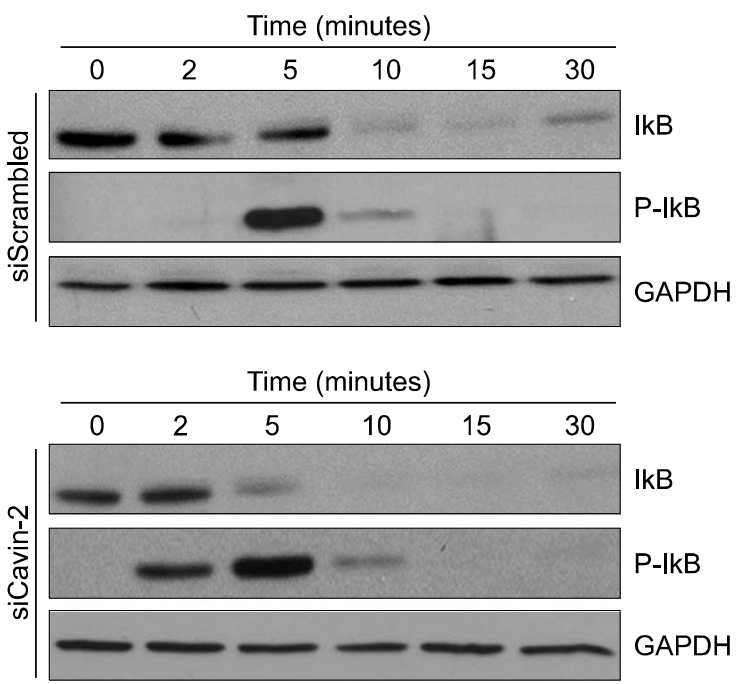

B

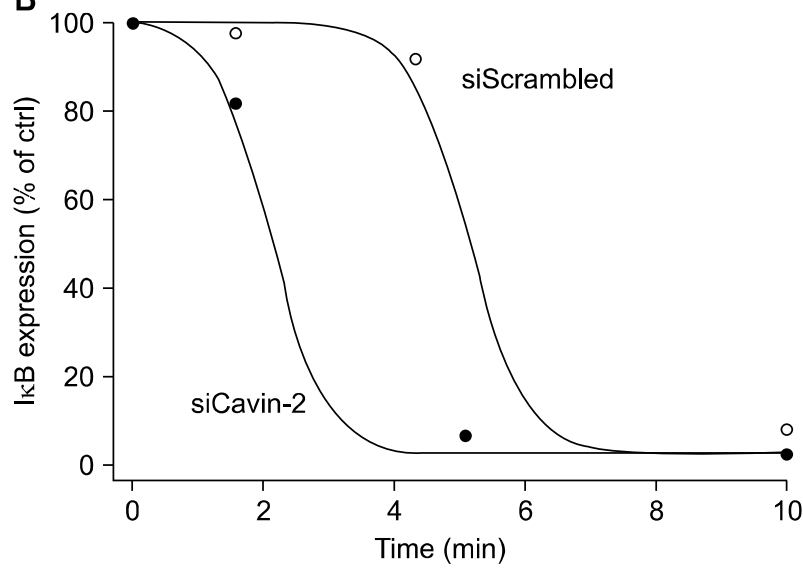

Fig. 5. TNF-mediated NF- $\kappa \mathrm{B}$ signaling is sustained upon Cavin-2 gene silencing. MSC were transiently transfected with a scrambled siRNA sequence, or with a specific siRNA against Cavin-2 (siCavin-2). Cells were then treated with $100 \mu \mathrm{M}$ TNF for the indicated time and (A) $\mid \kappa \mathrm{B}$, phosphorylated $\mathrm{I} \kappa \mathrm{B}$, and $\mathrm{GAPDH}$ expression were immunodetected in lysates, as described in the Methods section, and (B) quantified by scanning densitometry.

siScrambled or siCavin-2, then treated with TNF and lysates were harvested at the indicated times. We found that TNF effectively triggered $\mathrm{I} \kappa \mathrm{B}$ phosphorylation at $5 \mathrm{mi}-$ nutes, and that this subsequently led to $\mathrm{I} \kappa \mathrm{B}$ degradation as expected (Fig. 5A, upper panels). In contrast, Cavin-2 silencing resulted in a more rapid $(<5 \mathrm{~min})$ and sustained $\mathrm{I} \kappa \mathrm{B}$ phosphorylation (Fig. $5 \mathrm{~A}$, lower panels) which, in turn, also led to a rapid decrease in $\mathrm{I} \kappa \mathrm{B}$ (Fig. $5 \mathrm{~B}$ ). This data suggests that increased translocation to the nucleus of the freed p50 and p60 NF- $\kappa \mathrm{B}$ subunits may lead to sustained and/or increased COX-2 transcription and protein expression, as well as to increased MSC migration.

\section{Discussion}

Assessing the in vitro migration and tubulogenesis of MSC generally requires strategies to prime the cells, such as in response to serum-derived growth factors or to specific cytokines (33). Given the lack of in vitro inflammatory-like microenvironment components or extracellular growth factor-mediated cues, treatment with $\mathrm{TNF}-\alpha$ was used here to mimic and trigger a pro-inflammatory response. While TNF signal transducing events are, in part, believed to take place through caveolar components (34), neither the contribution of Cavins nor the balance in their stimulatory/repressive functions in inflammation and angiogenesis had yet been assessed in MSC.

Our current study specifically provides new insight into the inflammatory-suppressive functions of Cavin-2. While Cavin-1 and Cavin-3 expression are dose-dependently induced by $\mathrm{TNF}$, their individual silencing did not alter TNF-induced MSC migration nor COX-2 expression. In agreement with this, Cavin-1 and Cavin-3 expression were also induced by TNF in airway smooth muscle cells (35). In contrast, we found that only endogenous Cavin-2 expression is decreased upon TNF treatment, while its silencing led to amplified TNF-mediated MSC migration, tubulogenesis, and COX-2 expression. Silencing of Cavin-2 expression was, in fact, shown to alter the NF- $\kappa \mathrm{B}$ pathway, partially explaining the TNF- $\alpha$ effects. Collectively, we conclude that Cavin-2 exerts a suppressive role against the pro-inflammatory action of TNF since its silencing leads to increased TNF effects. Cavin-2 affects the formation of large caveolae, and accumulating evidence suggests that it is present in many cellular types; however, its clinical relevance remains debated. Whether Cavin-2 alters MSC tumour-homing ability in vivo remains unexplored, but opens up the possibility of investigating and possibly optimizing genetically-modified MSC-mediated targeted delivery of anti-cancer genes to tumours. Whether any regulation is exerted by Caveolin-1 on the repressive functions of Cavin-2 also remains to be investigated. Future studies will aim at defining whether Cavin-2 functions are caveolae-dependent or independent.

Cavin-2 was recently suggested to regulate Caveolin-1 expression, leading to slow oral squamous cell carcinoma (OSCC) proliferation by inactivation of the extracellular regulated kinase (ERK) pathway (36). Cavin-2 may therefore be a possible key regulator of OSCC progression via a Cavin-2/Caveolin-1/ERK pathway and a potential therapeutic target for developing new treatments for OSCCs. Further investigations on the roles of Cavin-2 will be required in inflammatory and angiogenic settings. Metabolic 
effects may also be expected upon modulation of Cavin-2 expression. Indeed, hypoxia was recently shown to inhibit Cavin-2 expression in adipocytes and to alter caveolar formation (37). This led to the inhibition of insulin signaling and the establishment of insulin resistance. Interestingly, downregulation of Cavin-2 upon hypoxia (37) correlates with increased hypoxic MSC endogenous tubulogenesis (31). The combined hypoxic and TNF-mediated inflammatory cues, which both led to decreased Cavin-2 expression, again reinforces the fact that Cavin-2 exerts a repressive control upon the angiogenic responses of a given cell model and within conditions approximating those found within the solid tumour microenvironment. While Cavin-1 expression is thought to be induced by TNF in MSC (our study), its regulation by hypoxia has yet to be assessed. On the other hand, Cavin-2 expression was found decreased upon hypoxia in 3T3 L1 adipocytes, that of Cavin-1 was also found decreased (37). How Cavin-1 is regulated in MSC upon hypoxia, and how this controls 3D-tubulogenesis is currently unknown. Although speculative, one can only safely envision at this point of our knowledge specific contributions of all Cavins in response to inflammatory and hypoxic cues which are expected to vary from a cell model to another. Further studies on each of Cavins' promoter sequences will be needed and will allow us to understand their transcriptional control.

In the current study, Cavin-1 and Cavin-3 were upregulated in MSC upon TNF treatment, suggesting that this positively correlates with the increased angiogenic phenotype of MSC upon pro-inflammatory stimulation. Interestingly, Cavin-3 transcript levels were also found to be induced by an alternate pro-inflammatory agent such as the phorbol ester PMA, and to regulate the expression of the biomarker MMP-9 in a human fibrosarcoma cell line (38). In that model, PMA was used to prime cell migration (39), and to increase secretion of MMP-9 (38), the secretion of which is generally correlated with inflammation, pro-angiogenic and metastatic processes (40). Such observations regarding the differential involvement of Cavins upon pro-inflammatory cues, whether these are triggered through receptor-mediated processes (TNF/TNFR) or through receptor-independent processes (PMA), suggest that pro-inflammatory and pro-angiogenic conditions can be effectively regulated by caveolar components. Through its capacity to repress TNF-mediated signaling, we have shown here the ability of Cavin-2 to regulate MSC migration and tubulogenesis. Given that MMP-9 was not inducible by TNF in MSC (not shown), the involvement of Cavins in MMP-9 regulation in that model remains to be established.

In summary, our study provides a better understanding of roles for the Cavin family members in regulating the angiogenic and inflammatory phenotype of MSC. Given the recognized contribution of MSC within (patho)physiological settings is currently of great interest towards either their therapeutic applications or as intermediates in solid tumour development, the dual roles of Caveolins and of Cavins must be better documented. Our study defines Cavin-2 as a putative suppressor regulator in TNF-mediated pro-inflammatory and pro-angiogenic conditions, and supports the recent evidence found in OSCC where its down-regulation was functionally and clinically linked to tumoural progression (36).

\section{Acknowledgements}

This study was funded by grant \#288249 from the Natural Sciences and Engineering Research Council of Canada (NSERC) and by the Institutional Research Chair in Cancer Prevention and Treatment held by Dr. Borhane Annabi at UQAM.

\section{Potential conflict of interest}

The authors have no conflicting financial interest.

\section{References}

1. Prockop DJ. Marrow stromal cells as stem cells for nonhematopoietic tissues. Science 1997;276:71-74

2. Horwitz EM, Le Blanc K, Dominici M, Mueller I, Slaper-Cortenbach I, Marini FC, Deans RJ, Krause DS, Keating A; International Society for Cellular Therapy. Clarification of the nomenclature for MSC: The International Society for Cellular Therapy position statement. Cytotherapy 2005;7:393-395

3. Studeny M, Marini FC, Champlin RE, Zompetta C, Fidler IJ, Andreeff M. Bone marrow-derived mesenchymal stem cells as vehicles for interferon-beta delivery into tumors. Cancer Res 2002;62:3603-3608

4. Nakamizo A, Marini F, Amano T, Khan A, Studeny M, Gumin J, Chen J, Hentschel S, Vecil G, Dembinski J, Andreeff M, Lang FF. Human bone marrow-derived mesenchymal stem cells in the treatment of gliomas. Cancer Res 2005;65:3307-3318

5. Annabi B, Naud E, Lee YT, Eliopoulos N, Galipeau J. Vascular progenitors derived from murine bone marrow stromal cells are regulated by fibroblast growth factor and are avidly recruited by vascularizing tumors. J Cell Biochem 2004;91:1146-1158

6. Birnbaum T, Roider J, Schankin CJ, Padovan CS, Schichor C, Goldbrunner R, Straube A. Malignant gliomas actively recruit bone marrow stromal cells by secreting angiogenic cytokines. J Neurooncol 2007;83:241-247

7. Dwyer RM, Potter-Beirne SM, Harrington KA, Lowery AJ, Hennessy E, Murphy JM, Barry FP, O'Brien T, Kerin MJ. 
Monocyte chemotactic protein-1 secreted by primary breast tumors stimulates migration of mesenchymal stem cells. Clin Cancer Res 2007;13:5020-5027

8. Djouad F, Plence P, Bony C, Tropel P, Apparailly F, Sany J, Noël D, Jorgensen C. Immunosuppressive effect of mesenchymal stem cells favors tumor growth in allogeneic animals. Blood 2003;102:3837-3844

9. Wang HH, Cui YL, Zaorsky NG, Lan J, Deng L, Zeng XL, Wu ZQ, Tao Z, Guo WH, Wang QX, Zhao LJ, Yuan ZY, Lu Y, Wang P, Meng MB. Mesenchymal stem cells generate pericytes to promote tumor recurrence via vasculogenesis after stereotactic body radiation therapy. Cancer Lett 2016;375:349-359

10. Suzuki K, Sun R, Origuchi M, Kanehira M, Takahata T, Itoh J, Umezawa A, Kijima H, Fukuda S, Saijo Y. Mesenchymal stromal cells promote tumor growth through the enhancement of neovascularization. Mol Med 2011;17: 579-587

11. Nassiri SM, Rahbarghazi R. Interactions of mesenchymal stem cells with endothelial cells. Stem Cells Dev 2014;23: 319-332

12. Carver LA, Schnitzer JE. Caveolae: mining little caves for new cancer targets. Nat Rev Cancer 2003;3:571-581

13. Shaul PW, Anderson RG. Role of plasmalemmal caveolae in signal transduction. Am J Physiol 1998;275:L843-L851

14. Parton RG, Simons K. The multiple faces of caveolae. Nat Rev Mol Cell Biol 2007;8:185-194

15. Gómez-González B, Escobar A. Altered functional development of the blood-brain barrier after early life stress in the rat. Brain Res Bull 2009;79:376-387

16. Baker N, Tuan RS. The less-often-traveled surface of stem cells: caveolin-1 and caveolae in stem cells, tissue repair and regeneration. Stem Cell Res Ther 2013;4:90

17. Senetta R, Stella G, Pozzi E, Sturli N, Massi D, Cassoni P. Caveolin-1 as a promoter of tumour spreading: when, how, where and why. J Cell Mol Med 2013;17:325-336

18. Massey KA, Schnitzer JE. Caveolae and cancer. Recent Results Cancer Res 2010;180:217-231

19. Baker N, Sohn J, Tuan RS. Promotion of human mesenchymal stem cell osteogenesis by PI3-kinase/Akt signaling, and the influence of caveolin-1/cholesterol homeostasis. Stem Cell Res Ther 2015;6:238

20. Baker N, Zhang G, You Y, Tuan RS. Caveolin-1 regulates proliferation and osteogenic differentiation of human mesenchymal stem cells. J Cell Biochem 2012;113:3773-3787

21. Parton RG, del Pozo MA. Caveolae as plasma membrane sensors, protectors and organizers. Nat Rev Mol Cell Biol 2013;14:98-112

22. Nassar ZD, Parat MO. Cavin family: new players in the biology of caveolae. Int Rev Cell Mol Biol 2015;320:235-305

23. Kovtun O, Tillu VA, Ariotti N, Parton RG, Collins BM. Cavin family proteins and the assembly of caveolae. J Cell Sci 2015;128:1269-1278

24. Aung CS, Hill MM, Bastiani M, Parton RG, Parat MO. PTRF-cavin-1 expression decreases the migration of PC3 prostate cancer cells: role of matrix metalloprotease 9. Eur
J Cell Biol 2011;90:136-142

25. Van den Steen PE, Dubois B, Nelissen I, Rudd PM, Dwek RA, Opdenakker G. Biochemistry and molecular biology of gelatinase B or matrix metalloproteinase-9 (MMP-9). Crit Rev Biochem Mol Biol 2002;37:375-536

26. Gupta R, Toufaily C, Annabi B. Caveolin and cavin family members: dual roles in cancer. Biochimie 2014;107:188-202

27. Chaudhary N, Gomez GA, Howes MT, Lo HP, McMahon KA, Rae JA, Schieber NL, Hill MM, Gaus K, Yap AS, Parton RG. Endocytic crosstalk: cavins, caveolins, and caveolae regulate clathrin-independent endocytosis. PLoS Biol 2014;12:e1001832

28. McMahon KA, Zajicek H, Li WP, Peyton MJ, Minna JD, Hernandez VJ, Luby-Phelps K, Anderson RG. SRBC/cavin-3 is a caveolin adapter protein that regulates caveolae function. EMBO J 2009;28:1001-1015

29. Bastiani M, Liu L, Hill MM, Jedrychowski MP, Nixon SJ, Lo HP, Abankwa D, Luetterforst R, Fernandez-Rojo M, Breen MR, Gygi SP, Vinten J, Walser PJ, North KN, Hancock JF, Pilch PF, Parton RG. MURC/Cavin-4 and cavin family members form tissue-specific caveolar complexes. J Cell Biol 2009;185:1259-1273

30. Yuan K, Huang C, Fox J, Gaid M, Weaver A, Li G, Singh $\mathrm{BB}, \mathrm{Gao} \mathrm{H}$, Wu M. Elevated inflammatory response in caveolin-1-deficient mice with Pseudomonas aeruginosa infection is mediated by STAT3 protein and nuclear factor kappaB (NF-kappaB). J Biol Chem 2011;286:21814-21825

31. Annabi B, Lee YT, Turcotte S, Naud E, Desrosiers RR, Champagne M, Eliopoulos N, Galipeau J, Béliveau R. Hypoxia promotes murine bone-marrow-derived stromal cell migration and tube formation. Stem Cells 2003;21: 337-347

32. Annabi B, Thibeault S, Lee YT, Bousquet-Gagnon N, Eliopoulos N, Barrette S, Galipeau J, Béliveau R. Matrix metalloproteinase regulation of sphingosine-1-phosphate-induced angiogenic properties of bone marrow stromal cells. Exp Hematol 2003;31:640-649

33. Jones GE. Cellular signaling in macrophage migration and chemotaxis. J Leukoc Biol 2000;68:593-602

34. D'Alessio A, Al-Lamki RS, Bradley JR, Pober JS. Caveolae participate in tumor necrosis factor receptor 1 signaling and internalization in a human endothelial cell line. Am J Pathol 2005;166:1273-1282

35. Sathish V, Thompson MA, Sinha S, Sieck GC, Prakash YS, Pabelick CM. Inflammation, caveolae and CD38-mediated calcium regulation in human airway smooth muscle. Biochim Biophys Acta 2014;1843:346-351

36. Unozawa M, Kasamatsu A, Higo M, Fukumoto C, Koyama T, Sakazume T, Nakashima D, Ogawara K, Yokoe H, Shiiba M, Tanzawa H, Uzawa K. Cavin-2 in oral cancer: A potential predictor for tumor progression. Mol Carcinog 2016;55:1037-1047

37. Regazzetti C, Dumas K, Lacas-Gervais S, Pastor F, Peraldi P, Bonnafous S, Dugail I, Le Lay S, Valet P, Le MarchandBrustel Y, Tran A, Gual P, Tanti JF, Cormont M, Giorgetti-Peraldi S. Hypoxia inhibits Cavin-1 and Cavin-2 
expression and down-regulates caveolae in adipocytes. Endocrinology 2015;156:789-801

38. Toufaily C, Charfi C, Annabi B, Annabi B. A role for the cavin-3/matrix metalloproteinase- 9 signaling axis in the regulation of PMA-activated human HT1080 fibrosarcoma cell neoplastic phenotype. Cancer Growth Metastasis 2014;

\section{7:43-51}

39. Gabler WL, Bullock WW, Creamer HR. Phorbol myristate acetate induction of chemotactic migration of human polymorphonuclear neutrophils. Inflammation 1993;17:521-530

40. McCawley LJ, Matrisian LM. Tumor progression: defining the soil round the tumor seed. Curr Biol 2001;11:R25-R27 